\title{
Catéter subcutáneo para la administración de heparina de bajo peso molecular en pediatría
}

\author{
ROXANA MÁRQUEZ N. ${ }^{1}$, PAOLA PINO A. ${ }^{1}$, PAULA FAJURI M. ${ }^{1}$, PAMELA ZÚÑIGA C. ${ }^{1}$ \\ 1. Servicio de Pediatria, Unidad de Paciente Crítico Pediátrica y Unidad de Hematología-Oncología. \\ Facultad de Medicina. Pontificia Universidad Católica de Chile.
}

\section{ABSTRACT \\ Subcutaneous catheter used for administration of low-molecular-weight-heparin in pe- diatrics}

Introduction: Subcutaneous catheter is a device easy to assemble, which was created for the administration of insulin in diabetic patients, especially in children, aiming to reduce the number of punctures, pain and anxiety of patients and their parents. Objective: To describe the experience using the catheter for subcutaneous administration of low molecular weight heparin (LMWH) in hospitalized pediatric patients. Patients and Method: A prospective descriptive study in 28 patients who used 87 subcutaneous catheters for the administration of LMWH in the Pediatric Service of the Universidad Catolica de Chile between July, 2010 and November, 2011. Results: Complications associated with the catheter had an incidence of $33 \%$ in total catheters evaluated; the most frequent complication was the presence of hematoma at the site of insertion $(26 \%)$. These complications occurred more frequently in male (38\% versus $31 \%$ in females) and younger patients ( 9 months versus 12 months), who received dalteparin (54\% versus 30\% of other types of heparin) administered every 24 hours (41\% versus 30\%, administered every 12 hours), and when the catheter was located on both thighs (36\% versus $32 \%$ in both arms); however, these differences were not statistically confirmed. Conclusion: The subcutaneous catheter is a good technique to be considered for LMWH in children as it presents minor complications for drug administration.

(Key words: Subcutaneous Catheter, low molecular weight heparin, enoxaparin, dalteparin, Pediatrics). Rev Chil Pediatr 2014; 85 (1): 46-51

\section{RESUMEN}

Introducción: El catéter subcutáneo es un dispositivo de fácil instalación, el cual fue ideado para la administración de insulinas en pacientes diabéticos, especialmente en pediatría, con el fin de disminuir el número de punciones, el dolor y la ansiedad de los pacientes y sus padres. Objetivo: Describir la experiencia del uso del catéter subcutáneo para la administración de heparina de bajo peso molecular (HBPM) en pacientes pediátri-

Recibido el 14 de enero de 2013, devuelto para corregir el 28 de abril de 2013, segunda versión 14 de julio de 2013, aceptado para publicación el 8 de octubre de 2013.

Este trabajo cumple con los requisitos sobre consentimiento /asentimiento informado, comité de ética, financiamiento, estudios animales y sobre la ausencia de conflictos de intereses según corresponda.

Correspondencia a:

Roxana Márquez N.

E-mail: roxmarq@med.puc.cl 
cos hospitalizados. Pacientes y Método: Estudio descriptivo prospectivo en 28 pacientes que utilizaron 87 catéteres subcutáneos instalados para la administración de HBPM, en el Servicio de Pediatría de la Pontificia Universidad Católica de Chile en el período comprendido entre los meses de julio de 2010 y noviembre de 2011. Resultados: Las complicaciones asociadas al catéter presentaron una incidencia de $33 \%$ en el total de catéteres evaluados, siendo la más frecuente la presencia de hematoma en el sitio de inserción (26\%). Estas complicaciones se presentaron con mayor frecuencia en pacientes de sexo masculino (38\% versus $31 \%$ en sexo femenino) de menor edad ( 9 meses versus 12 meses), con indicación de dalteparina ( $54 \%$ versus $30 \%$ con otros tipos de heparina) administrada cada $24 \mathrm{~h}$ ( $41 \%$ versus $30 \%$ cuando fue administrada cada $12 \mathrm{~h}$ ), y cuando el catéter estuvo ubicado en ambos muslos (36\% versus $32 \%$ en ambos brazos); sin embargo, estas diferencias no fueron confirmadas estadísticamente. Conclusión: El catéter subcutáneo es una buena técnica a considerar para la administración de HBPM en la edad pediátrica, ya que permite la administración del medicamento con complicaciones leves asociadas a su uso.

(Palabras clave: Catéter subcutáneo; Heparina de bajo peso molecular; Enoxaparina; Dalteparina; Pediatría). Rev Chil Pediatr 2014; 85 (1): 46-51

\section{Introducción}

Dentro de la población pediátrica los eventos tromboembólicos son frecuentes, sobretodo en pacientes recién nacidos ${ }^{1-5}$ que han sido hospitalizados en servicios de cuidados intensivos y sometidos a una mayor utilización de catéteres venosos centrales ${ }^{1-6}$.

Debido a sus múltiples ventajas ${ }^{7}$, la heparina de bajo peso molecular (HBPM) se ha convertido en una terapia de elección tanto para el tratamiento como la profilaxis de enfermedades tromboembólicas ${ }^{4,6-8}$, sustituyendo en muchos casos a la heparina no fraccionada. Dentro de sus principales beneficios están la facilidad en su administración, gran eficacia, menor riesgo de trombocitopenia, menor sangramiento, menor necesidad de monitorización, efecto predecible y menor número de punciones evitando dolor asociado ${ }^{1-3}$. Sin embargo, no está exenta de algunos riesgos como son equimosis o hematomas superficiales, necrosis de piel y de tejido subcutáneo ${ }^{9,10}$.

El catéter de inserción subcutánea es un dispositivo de fácil instalación, el cual fue ideado para la administración de insulinas en pacientes diabéticos, especialmente en pediatría, con el fin de disminuir el número de punciones, el dolor y la ansiedad de los pacientes y sus padres ${ }^{1,3,11-14}$. En los últimos años, se ha ampliado su uso, utilizándose para la administración de medicamentos oncológicos ${ }^{15,16}$, morfina ${ }^{17}$ y enoxaparina para pacientes con enfermedades tromboembólicas ${ }^{1-3,6,8,18,19}$, entre otros.
Existen publicaciones a nivel internacional acerca del uso de este catéter para la administración de insulina y de otros medicamentos en pacientes pediátricos. Estos reportan buenos resultados en relación a la disminución del dolor y la ansiedad asociados al menor número de punciones y a la presencia de complicaciones leves ${ }^{1,3,11-14}$.

En Chile, existen centros tanto públicos como privados que utilizan este dispositivo como alternativa para la administración de medicamentos subcutáneos. No obstante, no se han encontrado publicaciones chilenas respecto al uso de este catéter para la administración de HBPM ni de otros medicamentos, a excepción de publicaciones en relación a medicamentos administrados por punción subcutánea directa en pacientes recién nacidos ${ }^{20}$, en adultos $^{21}$ y embarazadas ${ }^{22}$.

El objetivo de este estudio es describir la experiencia del uso del catéter subcutáneo para la administración de HBPM en pacientes pediátricos hospitalizados.

\section{Pacientes y Método}

Estudio descriptivo prospectivo, donde se realizó seguimiento a todos los pacientes con catéteres subcutáneos instalados para la administración de HBPM, en el Servicio de Pediatría de la Pontificia Universidad Católica de Chile en el período comprendido entre el mes de julio de 2010 y noviembre de 2011, correspondiendo a un universo de 103 catéteres. 
Los catéteres subcutáneos utilizados correspondieron a la marca comercial Insuflón $\AA$, los cuales fueron instalados por enfermeras del servicio sin sedación ni analgesia durante el procedimiento, utilizando la técnica de inyección subcutánea descrita por el fabricante (tabla 1). Se excluyeron de este análisis los catéteres de pacientes que fueron trasladados a otro centro hospitalario u otro servicio, o dados de alta con el catéter para continuación de tratamiento en el hogar, ya que no fue posible realizar el seguimiento $(n=16)$.

Las variables consideradas en este estudio fueron: características del niño (sexo, edad y diagnóstico de ingreso); características del medicamento administrado (tipo de heparina indicada, motivo de indicación y frecuencia de administración); características del catéter (número de catéteres por niño, ubicación del catéter, días de permanencia y motivo de retiro del catéter); complicaciones asociadas al uso del catéter (hematoma, sangramiento, induración, desplazamiento y retiro accidental) y día de aparición de las complicaciones; comparación entre la incidencia de complicaciones y las variables sexo y edad del paciente, tipo de heparina indicada, frecuencia de administración, días de permanencia y ubicación del catéter.

El seguimiento sistemático de estos catéteres se realizó en forma diaria utilizando una hoja de registro interna diseñada para tal efecto. Cabe destacar que en este estudio no fue evaluada la presencia de dolor durante la instalación del dispositivo.

Para el análisis de las variables se utilizó el programa SPSS 15.0. Se realizaron medidas de tendencia central y dispersión y análisis de frecuencia.

Este estudio fue aprobado por el Comité de Ética del Hospital Clínico UC con dispensa de consentimiento informado, dado que los datos fueron levantados desde los registros internos del Servicio de Pediatría del Hospital Clínico UC y traspasados a una base de datos anónima, sin la identificación de los pacientes involucrados.

\section{Resultados}

Durante el período de seguimiento fueron estudiados 87 catéteres de 28 pacientes. Del total de pacientes, la mayoría correspondía a sexo masculino $(\mathrm{n}=16)$. La edad de los niños presentó un rango entre 1 y 183 meses, con una mediana de 22,5 meses. El diagnóstico de ingreso más frecuente fue la enfermedad cardiovascular $(\mathrm{n}=14)$, seguido por enfermedad renal $(n=6)$, gastrointestinal $(n=3)$ y otros diagnósticos como enfermedad neurológica, broncopulmonar, metabólica e inmunológica $(\mathrm{n}=5)$.

En el total de catéteres instalados, el tipo de heparina indicada con mayor frecuencia fue enoxaparina (87\%) y el resto dalteparina. Entre los motivos de indicación se encontraron: tratamiento de enfermedad trombótica (46\%), profilaxis de enfermedad tromboembólica $(33 \%)$ y terapia mixta (tratamiento y profilaxis) (21\%). Según la frecuencia de administración, la mayoría de los pacientes recibió HBPM cada 12 h (66\%), seguido de cada $24 \mathrm{~h}$ $(33 \%)$ y en un paciente, cada $8 \mathrm{~h}$.

\section{Tabla 1. Pasos para la instalación del catéter subcutáneo}

\begin{tabular}{|cl|}
\hline Pasos & Descripción \\
1 & Remover el protector de la aguja \\
2 & Escoger el sitio de punción e inserte en la piel plegada con sus dedos, en un ángulo de $45^{\circ}$ \\
3 & Retirar el introductor de aguja \\
4 & Retirar la parte frontal de la cobertura del apósito adhesivo \\
5 & $\begin{array}{l}\text { Poner el apósito sobre el catéter, asegurándose que el sitio de inserción quede visible por la ventana del apósito y } \\
\text { remover la parte trasera de la cobertura }\end{array}$ \\
6 & Comprimir cuidadosamente la zona del adhesivo \\
\hline
\end{tabular}


El número de catéteres utilizados por niño presentó un rango entre 1 a 18 catéteres, con una mediana de 2 catéteres por niño. La ubicación del catéter utilizada con más frecuencia fueron ambos brazos con un 68\% (tabla 2). Los días de permanencia presentaron un rango entre 1 a 7 días, con una mediana de 5 días. El motivo de retiro más frecuente fue por cambio programado (tabla 3 ).

Las complicaciones asociadas al catéter presentaron una incidencia de $33 \%$ en el total de catéteres evaluados. La complicación más frecuente fue hematoma en el sitio de inserción $(26 \%)$, seguido de induración (12\%) y sangra-

Tabla 2. Distribución porcentual según ubicación de catéter subcutáneo

\begin{tabular}{|lcc|}
\hline Ubicación del catéter & Frecuencia & Porcentaje \\
\hline Brazo derecho & 31 & 36 \\
\hline Brazo izquierdo & 28 & 32 \\
\hline Muslo derecho & 16 & 18 \\
Muslo izquierdo & 12 & 14 \\
\hline Total & 87 & 100 \\
\hline
\end{tabular}

miento $(7 \%)$, existiendo más de una complicación en algunos catéteres. No se reportaron desplazamiento ni retiro accidental. El día de aparición de las complicaciones presentó un rango entre 1 a 5 días, con una mediana de 3 días.

La comparación entre la incidencia de complicaciones y las variables estudiadas demostraron que existían leves diferencias en relación a la edad de los pacientes y a la duración del dispositivo (tabla 4). Así como también en relación al sexo del paciente, al tipo de heparina indicada, a la frecuencia de administración y a la ubicación del catéter (tabla 5).

Tabla 3. Distribución porcentual según motivo de retiro del catéter

\begin{tabular}{|lcc|}
\hline Motivo de retiro & Frecuencia & Porcentaje \\
\hline Cambio programado & 40 & 46 \\
\hline Complicaciones & 29 & 33 \\
\hline Suspensión del tratamiento & 14 & 16 \\
\hline Alta del paciente & 4 & 5 \\
\hline Total & 87 & 100 \\
\hline
\end{tabular}

Tabla 4. Comparación entre la incidencia de complicaciones y la edad del paciente y la duración del catéter

\begin{tabular}{|llcc|}
\hline Variables evaluadas & $\begin{array}{c}\text { Sin complicaciones } \\
\mathbf{n = 5 8}\end{array}$ & $\begin{array}{c}\text { Con complicaciones } \\
\mathbf{n}=\mathbf{2 9}\end{array}$ \\
\hline Edad del paciente & Mediana & 12,5 meses & 9 meses \\
& Rango & $(1-183$ meses $)$ & $(1-183$ meses $)$ \\
Duración del catéter & Mediana & 5 días & 4 días \\
& Rango & $(1-7$ días $)$ & $(1-6$ días $)$ \\
\hline
\end{tabular}

Tabla 5. Comparación entre la incidencia de complicaciones y el sexo del paciente, el tipo de heparina indicada, la frecuencia de administración y la ubicación del catéter

\begin{tabular}{|llcc|}
\hline Variables evaluadas & & $\begin{array}{c}\text { \% sin complicaciones } \\
\mathbf{n = 5 8}\end{array}$ & $\begin{array}{c}\text { \% con complicaciones } \\
\mathbf{n}=\mathbf{2 9}\end{array}$ \\
\hline Sexo del paciente & Masculino & 69 & 31 \\
& Femenino & 62 & 38 \\
Tipo de heparina indicada & Enoxiparina & 70 & 30 \\
& Dalteparina & 46 & 54 \\
\hline Frecuencia de administración & Cada 12 h & 70 & 30 \\
\cline { 2 - 2 } & Cada 24 h & 59 & 41 \\
& Ambos brazos & 68 & 32 \\
\hline
\end{tabular}




\section{Discusión}

En este estudio, un tercio de los participantes estudiados presentó complicaciones asociadas al uso de catéter subcutáneo. Estas complicaciones se presentaron con mayor frecuencia en pacientes de sexo masculino (38\% versus $31 \%$ ) de menor edad (9 meses versus 12 meses), con indicación de dalteparina (54\% versus 30\%) administrada cada $24 \mathrm{~h}$ (41\% versus $30 \%$ ) cuando era administrada cada $12 \mathrm{~h}$, y cuando el catéter estuvo ubicado en ambos muslos (36\% versus $32 \%$ en ambos brazos); sin embargo, estas diferencias no fueron confirmadas estadísticamente. No se encontró en la literatura estudios que respalden estos hallazgos.

La complicación asociada al uso de catéter que se presenta con mayor frecuencia tanto en el presente estudio como en la literatura, es la aparición de hematomas en el sitio de inserción con una incidencia de 0 a $56 \%$ 1,3,9,10,14,18. complicación que también se observa en pacientes con administración de HBPM por vía subcutánea directa con una incidencia de 47 a $100 \%{ }^{9-10,19}$

Según la literatura, los hematomas en el sitio de inserción se presentan principalmente en pacientes que reciben tratamiento anticoagulante a través del dispositivo subcutáneo ${ }^{1-3,6,8,18}$. En cambio, en pacientes que reciben insulina esta complicación presentó una incidencia de sólo un $0,8 \%$, reportando otras complicaciones como enrojecimiento $(5,6$ a $15 \%)$, eczema $(1,7$ a $27 \%)$ y lipohipertrofia $(2,4 \text { a } 29 \%)^{11,13-14}$; mientras que los que reciben G-CSF y morfina sólo presentaron aumento de volumen $(2 \%)$, sangramiento (0 a $5 \%$ ) y enrojecimiento $(3 \%)^{16-18}$. También se han reportado algunas complicaciones mayores asociadas al uso de HBPM como sangramiento mayor en un 0,7 a $5 \%$ de los $\operatorname{casos}^{3,4,7,8}$, un caso de infección local con necesidad de tratamiento antibiótico $^{1}$, y un hematoma gigante con síndrome compartimental en un paciente con diagnóstico de sepsis persistente ${ }^{18}$.

Dentro de las fortalezas del presente estudio se encuentra la baja variabilidad en el método de recolección de datos, dado que la pauta de cotejo fue aplicada sólo por las enfermeras investigadoras del estudio. Dentro de las limitaciones del estudio se encuentra que, los pacientes dados de alta con dicho catéter no fueron evaluados en forma programada. Sin embargo, todas las madres recibieron educación y consiguieron capacitarse en forma efectiva en la instalación de el dispositivo y en la administración del medicamento; además, todos los pacientes fueron controlados en la consulta médica del Policlínico de Hemostasia y Trombosis de la Red Salud UC, sin presentar complicaciones. Otra limitación sería no haber objetivado el dolor mediante las escalas establecidas; ya que, según algunos autores éste es uno de los principales motivos por el cual se indica el uso de catéter subcutáneo ${ }^{1,3,11-14}$.

En conclusión, el catéter subcutáneo es una buena técnica a considerar para la administración de HBPM en la edad pediátrica; ya que permite la administración del medicamento con complicaciones leves asociadas a su uso, y considerando además que, según la literatura, el uso de este dispositivo disminuye el dolor y ansiedad asociados a la punción $n^{1,3,11-14}$.

Esta investigación es la primera que se realiza en el Servicio de Pediatría en relación al uso de catéteres subcutáneos, constituyendo un aporte tanto para los pacientes como para el servicio. Creemos relevante continuar con esta línea de investigación, a través de estudios que comparen el uso del catéter con la punción subcutánea directa, considerando otras variables como: peso de los pacientes, cantidad de tejido subcutáneo de los pacientes, dosis administrada para alcanzar niveles terapéuticos, costos asociados, dolor y satisfacción de los pacientes y sus familias en relación al uso del catéter.

\section{Agradecimientos}

Al equipo de enfermeras que participó en la instalación de los catéteres subcutáneos. A la PhD María Teresa Urrutia, Profesora Asociada. Escuela de Enfermería UC; quien supervisó la redacción del manuscrito. 


\section{Referencias}

1.- Malowany J, Knoppert D, Chan A, Pepelassis D, Lee $D$ : Enoxaparin use in the neonatal intensive care unit: Experience over 8 years. Pharmacotherapy 2007; 27 (9): 1263-71.

2.- Massicotte P, Adams M, Marzinotto V, Brooker L, Andrew $M$ : Low-molecular-weight heparin in pediatric patients with thrombotic disease: a dose finding study. J Pediatr 1996; 128 (3): 313-8.

3.- Malowany J, Monagle P, Knoppert D, et al: Enoxaparin for neonatal thrombosis: A call for a higher dose for neonates. Thromb Res 2008; 122: 826-30.

4.- Streif W, Goebel G, Chan A, Massicotte M: Use of low molecular mass heparin (enoxaparin) in newborn infants: A prospective cohort study of 62 patients. Arch Dis Child Fetal Neonatal Ed. 2003; 88: 365-70.

5.- Michaels L, Gurian M, Hegyi T, Drachtman R: Low molecular weight heparin in the treatment of venous and arterial thromboses in the premature infant. Pediatrics 2004; 114 (3): 703-7.

6.- Sánchez J, Gunawardena S, Muñoz R, et al: Do neonates, infants and young children need a higher dose of enoxaparin in the cardiac intensive care unit? Cardiol Young 2010; 20 (2): 138-43.

7.- Nowak-Göttl U, Bidlingmaier C, Krümpel A, Göttl L, Kenet $G$ : Pharmacokinetics, efficacy, and safety of LMWHs in venous thrombosis and stroke in neonates, infants and children. Br J Pharmacol 2008; 153 (6): 1120-7.

8.- Ignjatovic V, Najid S, Newall F, Summerhayes R, Monagle $P$ : Dosing and monitoring of enoxiparin (Low molecular weight heparin) therapy in children. $\mathrm{Br} \mathrm{J}$ Haematol 2010; 149 (5): 734-8.

9.- Alcahúd C, Iglesias T, Lázaro C, et al: Administración de heparina de bajo peso molecular y aparición de complicaciones locales en pacientes de cardiología. Enferm Cardiol 2009; 16 (47-48): 94-8.

10.- Gómez M, Martínez M, García I: ¿Cuál es la técnica idónea para disminuir las complicaciones locales secundarias a la administración subcutánea de enoxaparina? Ensayo clínico aleatorizado. Enferm Clin 2005; 15 (6): 329-34.

11.- Hanas R, Adolfsson P, Elfin-Akesson K, et al: Indwe- lling catheters used from the onset of diabetes decrease injection pain and pre-injection anxiety. J Pediatr 2002; 140 (3): 315-20.

12.- Burdick P, Cooper S, Horner B, et al: Use of a subcutaneous injection port to improve glycemic control in children with type 1 diabetes. Pediatr Diabetes 2009; 10 (2): 116-9.

13.- Hanas $R$, Stanke C, Östberg H: Diagnosis of the cause of malfunction of indwelling catheters for insulin injections by the use of digital fluoroscopy. Pediatr Radiol 2000; 30 (10): 674-6.

14.- Hanas R: Reducing injection pain in children and adolescents with diabetes: A review of indwelling catheters. Pediatr Diabetes 2004; 5 (2): 102-11.

15.- De Jong M, Carbière T, Van de Heuvel-Eibrink M: The use of an insuflon device for the administration of G-CSF in pediatric cancer patients. Suport Care Cancer 2006; 14 (1): 98-100.

16.- Dyer S, Collins C, Baghurst P, Saxon B, Meachan B: Insuflon versus subcutaneous injection for cytokine administration in children and adolescents: a randomized crossover study. J Pediatr Oncol Nurs 2004; 21 (2): 79-86.

17.- Rouss K, Gerber A, Albisetti M, Hug M, Bernet V: Long-term subcutaneous morphine administration after surgery in newborns. J Perinat Med 2007; 35 (1): 79-81.

18.- Obaid L, Byrne P, Cheung P: Compartment syndrome in an ELBW infant receiving low-molecular-weight heparins. J Pediatr 2004; 144 (4): 549.

19.- O`Brien $S$, Lee $H$, Ritchey $K$ : Once-daily enoxaparin in pediatric thromboembolism: A dose finding and pharmacodynamics/farmacokinetics study. J Thromb Haemost 2007; 5 (9): 1985-7.

20.- Ladino M, Ruiz-Esquide F, Guardia S: Trombosis venosa profunda en relación a un catéter central. Edema neonatal en esclavina, un caso clínico. Rev Chil Pediatr 2001; 72 (4): 340-4.

21.- Conte G, Figueroa $G$ : Frecuencia de tromboembolismo venoso en pacientes hospitalizados con cáncer. Factores de riesgo y eficacia de la tromboprofilaxis farmacológica. Rev Med Chile 2008; 136 (12): 1528-34.

22.- Hasbún J, Conte G: Manejo de la embarazada con trombofilia hereditaria. Rev Chil Obstet Ginecol 2003; 68 (6): 458-70. 